\title{
Crystal Structures of the Precursor Form of Glucose-Fructose Oxidoreductase from Zymomonas mobilis and Its Complexes with Bound Ligands ${ }^{\dagger, 末}$
}

\author{
Didier Nurizzo, ${ }^{\S, \|}$ Dirk Halbig, ${ }^{\S, \perp}$ Georg A. Sprenger, ${ }^{\perp}$ and Edward N. Baker*,§,@ \\ School of Biological Sciences, University of Auckland, Auckland, New Zealand, Institut für Biotechnologie 1, \\ Forschungszentrum Jülich GmbH, D-52425 Jülich, Germany, and Department of Chemistry, University of Auckland, \\ Auckland, New Zealand \\ Received June 27, 2001; Revised Manuscript Received September 7, 2001
}

\begin{abstract}
The NADP(H)-dependent enzyme glucose-fructose oxidoreductase (GFOR) is a classic example of a redox protein that is translocated across a membrane in fully folded form. GFOR is synthesized in the cytoplasm with a 52-residue signal peptide, giving a precursor form, preGFOR, that is fully active and has its cofactor tightly bound. A twin-arginine motif in the signal peptide directs it to a Sec-independent pathway by which it is translocated, in fully folded form, into the periplasm where it functions to produce sorbitol for osmoprotection. We have determined the crystal structures of four different forms of preGFOR, (i) oxidized preGFOR, with succinate bound in the active site, (ii) oxidized preGFOR with glycerol bound, (iii) reduced preGFOR in $0.3 \mathrm{M}$ glucose, and (iv) reduced preGFOR in $1.5 \mathrm{M}$ sorbitol, at resolutions of $2.2,2.05,2.5$, and $2.6 \AA$, respectively. In all four crystal structures, the signal peptide is disordered, implying a flexibility that may be important for its interaction with the translocation apparatus; a factor contributing to this disorder may be the high positive charge of the protein surface in the region where the signal peptide emerges. This may disfavor a stable association between the signal peptide and the rest of the protein. The crystal structures show that the mature enzyme portion of preGFOR is identical to native GFOR, in structure and cofactor binding, explaining the enzymatic activity of the precursor form. In the glycerol complex, preGFOR gll, a bound glycerol molecule models the binding of the glucose substrate, with its $\mathrm{O} 1$ atom hydrogen bonded to the essential acid/base catalyst, Tyr269, and C1 only $3 \AA$ from C4

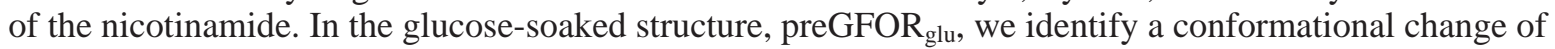
the nearby Lys 181 that probably results from the oxidation of glucose to gluconolactone, and functions to prevent rebinding of glucose prior to the binding of fructose. In this conformational change, the Lys181 side chain moves closer to the nicotinamide ring, stabilized by its increased negative charge.
\end{abstract}

Translocation of proteins across biological membranes to the cellular or extracellular locations that are appropriate to their activity is one of the most important processes in cell biology. One of the best understood mechanisms is the Secdependent pathway by which proteins are translocated across the inner membrane in Escherichia coli and other Gramnegative bacteria $(1,2)$. Proteins are targeted to this pathway by an N-terminal signal sequence of $\sim 20$ residues, and pass through the membrane in an unfolded state (3).

Other pathways are also beginning to be recognized, however. In particular, a radically different mechanism has

$\dagger$ This work was supported by an International Research Scholar award from the Howard Hughes Medical Institute (to E.N.B.), the Deutsche Forschungsgemeinschaft (Grant Sp503/1-4 to G.S.), the Deutsche Akademische Austauschdienst (postdoctoral fellowship to D.H.), and the Wellcome Trust (major equipment grant to E.N.B.).

$\doteqdot$ Atomic coordinates and structure factor amplitudes have been deposited with the Protein Data Bank, as entries 1h6a (preGFOR glu$_{\text {) }}$ ),

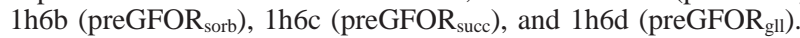

* To whom correspondence should be addressed. Phone: $(+64)$ 9-373-7599. Fax: (+64) 9-373-7619. E-mail: ted.baker@auckland.ac.nz.

$\S$ School of Biological Sciences, University of Auckland.

"Present address: Department of Chemistry, University of York, York YO10 5DD, United Kingdom.

${ }^{\perp}$ Forschungszentrum Jülich GmbH.

${ }^{\circledR}$ Department of Chemistry, University of Auckland. been identified that uses a transmembrane $\mathrm{pH}$ gradient to translocate proteins across the thylakoid membrane into plant chloroplasts $(4,5)$. A similar Sec-independent pathway also exists in Gram-negative bacteria, by which proteins synthesized in the cytoplasm may be translocated across the inner membrane into the periplasm. Proteins targeted to this pathway have a longer $\mathrm{N}$-terminal presequence, including a twin-arginine consensus motif (S/T-R-R-X-L-F-K) that resides between a polar $\mathrm{N}$-terminal region and a hydrophobic stretch $(6,7)$. Remarkably, proteins that use this pathway are translocated in fully folded form. Many of the substrate proteins for this pathway are in fact proteins with complex redox cofactors that are inserted in the cytoplasm prior to translocation into the periplasm $(6-8)$.

The enzyme glucose-fructose oxidoreductase $(\mathrm{GFOR})^{1}$ from the Gram-negative bacterium Zymomonas mobilis provides a well-characterized example of a protein that is translocated into the periplasm by this Sec-independent twinArg mechanism (9). The physiological role of GFOR is to

\footnotetext{
${ }^{1}$ Abbreviations: GFOR, glucose-fructose oxidoreductase; preGFOR, precursor form of glucose-fructose oxidoreductase, residues 1-433, with Arg30 and Arg31 mutated to Lys; G6PD, glucose-6-phosphate dehydrogenase; DD, dihydrodiol dehydrogenase; PEG, polyethylene glycol.
} 


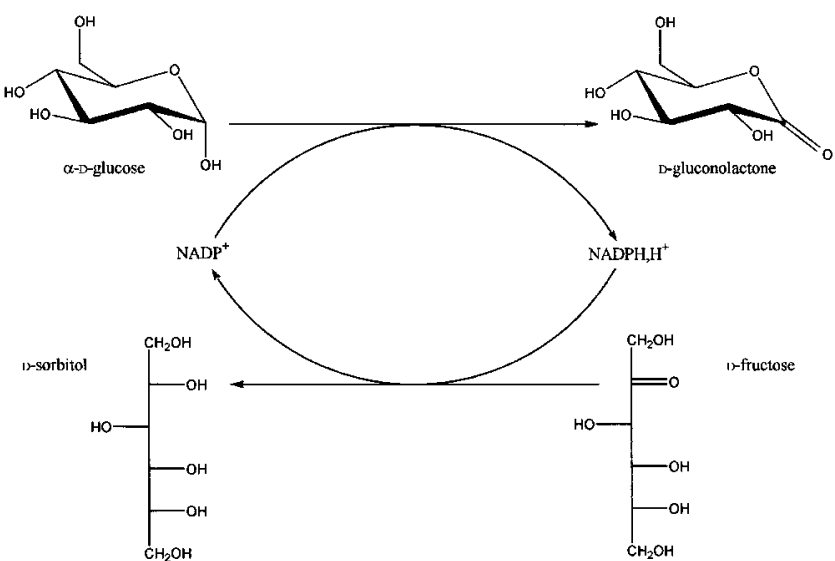

FIGURE 1: Reaction scheme for glucose-fructose oxidoreductase. The reaction takes place by a classic ping-pong mechanism (11, 12) in which oxidation of glucose to gluconolactone is followed by reduction of fructose to sorbitol, with the $\operatorname{NADP}(\mathrm{H})$, tightly bound to the apoprotein, acting as the redox carrier.

produce sorbitol in the periplasm, as a compatible solute that protects against osmotic stress when the bacterium grows in high concentrations of sugars (10). The NADP(H)-dependent reaction occurs by a ping-pong mechanism $(11,12)$ in which (i) glucose is oxidized by NADP to gluconolactone, after which (ii) the NADPH reduces fructose to sorbitol (Figure 1). The sorbitol is not further metabolized and accumulates in the cytosol as a counter to osmotic stress, whereas the gluconolactone is degraded via the Entner-Doudoroff pathway $(11,13)$.

GFOR is synthesized in the cytosol in an active precursor form termed preGFOR (14), with an N-terminal signal sequence of 52 amino acids $(15,16)$. This sequence includes a positively charged $\mathrm{N}$-terminal region (residues 1-32) followed by a hydrophobic core (residues 33-46) and a more polar C-terminal region (residues 47-52). Mutagenesis of one or both of the two residues that comprise the twinarginine motif, Arg30 and Arg31, shows that these residues are essential for translocation into the periplasm (9). Mutation of Lys121, which is involved in binding the $\operatorname{NADP}(\mathrm{H})$ cofactor, also severely affects translocation (9), suggesting that cofactor binding is also necessary for efficient translocation. This result also implies that the cofactor is inserted into preGFOR in the cytoplasm, and the protein is then translocated across the membrane in fully folded form. Processing of preGFOR to the mature enzyme occurs during transfer, by cleavage of an Ala-Ala bond (a common cleavage site for prokaryotic leader sequences) $(15,16)$.

The crystal structure of the mature form of GFOR has been determined previously at $2.7 \AA$ resolution (17). The molecule forms a tetramer in which each monomer [43 kDa, 381 amino acid residues (18)] is folded into two independent, closely associated domains. The $\mathrm{N}$-terminal domain has a classical dinucleotide-binding (Rossmann) $\alpha / \beta$-fold (19), and the C-terminal domain is based on a large "open-faced" (20) $\beta$-sheet of eight mostly antiparallel $\beta$-strands. The GFOR tetramer is a dimer of dimers, in which two monomers associate by stacking of their open-faced sheets, and tetramer formation is dependent on an N-terminal "arm", residues 5383 (residues $1-31$ of the mature enzyme) from each subunit that wraps around an adjacent subunit $(17,21)$. Extended arm exchanges of this type are known to contribute substantially to oligomer stability in other proteins $(22,23)$, and in GFOR deletion of the N-terminal arm (18) results in a dimer rather than a tetramer (21). In GFOR, however, the $\mathrm{N}$-terminal arm is also important for tight cofactor binding; the $\operatorname{NADP}(\mathrm{H})$ is located between the two domains of the monomer, with its adenyl portion buried beneath the $\mathrm{N}$ terminal arm extending from an adjacent subunit (17). This correlation between tetramer formation and cofactor binding implies that GFOR is likely to be translocated as a fully folded tetramer after insertion of the $\operatorname{NADP}(\mathrm{H})$.

A mutant precursor form of GFOR, in which residues Arg30 and Arg31 are replaced with lysine, can be purified and obtained in large amounts after expression of the respective mutant gfo gene in $Z$. mobilis, since translocation of preGFOR mutant R30K/R31K across the inner membrane is severely impaired and the protein consequently accumulates in the cytoplasm (9). The preGFOR so obtained has its $\mathrm{NADP}(\mathrm{H})$ cofactor bound and is enzymatically active (9). The availability of this precursor form in large quantities provides a unique opportunity to answer several questions relating to the translocation and activity of the enzyme. Does the 52-residue signal peptide of preGFOR have a defined three-dimensional structure, and if so, how does it associate with the mature enzyme segment and how does it present its consensus sequence motif for recognition by the translocation apparatus? How does the catalytic site of the precursor form compare with that of the mature enzyme, and is the $\mathrm{NADP}(\mathrm{H})$ cofactor similarly bound? How does GFOR bind its two substrates, glucose and fructose? Both substrates share a common binding site, which apparently can accommodate both the cyclic form of D-glucose (with a specificity for $\beta$-D-glucose) and the open chain form of D-fructose (11, 12). Neither the location of the binding site nor the mode of substrate binding is known, however.

Here we describe crystal structures of oxidized preGFOR R30K/R31K (termed hereafter preGFOR), in complexes with succinate $\left(\right.$ preGFOR $_{\text {succ }}$ ) and with glycerol $\left(\right.$ preGFOR $\left._{\mathrm{gll}}\right)$, at 2.2 and $2.05 \AA$ resolution, respectively, and of reduced preGFOR, after incubation with glucose $\left(\right.$ preGFOR $_{\text {glu }}$ ) and with sorbitol (preGFOR ${ }_{\text {sorb }}$ ), at 2.5 and $2.6 \AA$ resolution, respectively. Interestingly, in all cases the signal peptide extends into solvent channels in the crystal and lacks any clearly defined structure, implying that flexibility may be an important element in recognition and processing. On the other hand, the mature enzyme component of preGFOR is well-defined, and this series of structures, several at a resolution higher than that of wild-type GFOR (17), enables us to identify the likely binding site for sugar substrates and an intriguing conformational change that is implicated in the enzyme activity.

\section{MATERIALS AND METHODS}

Bacterial Strains, Plasmids, and Media. E. coli K-12 strain JM109 (24) was used for cloning and mutagenesis of the R30K/R31K gfo allele. For interspecies conjugation, E. coli S17-1 (25) was used. GFOR-deficient strain ACM3963 and the recombinant derivative thereof were maintained and cultivated anaerobically as described previously (18). Sitedirected mutagenesis and enzymatic measurements of GFOR enzyme activity were carried out as described elsewhere (9).

Expression and Purification. PreGFOR R30K/R31K mutant protein (preGFOR) was purified from the respective 


\begin{tabular}{|c|c|c|c|c|}
\hline & preGFOR $_{\text {succ }}$ & preGFOR $_{\mathrm{gll}}$ & preGFOR $_{\text {glu }}$ & preGFOR $_{\text {sorb }}$ \\
\hline space group & $P 2,2,2$ & $P 2_{1}$ & $P 2,2,2$ & $P 2,2,2$ \\
\hline unit cell lengths $(\AA)$ & $84.1,93.3,115.4$ & $115.7,83.8,279.2$ & $83.9,93.1,115.5$ & $83.8,92.8,115.5$ \\
\hline angles (deg) & $90,90,90$ & $90,90,90$ & $90,90,90$ & $90,90,90$ \\
\hline$V_{\mathrm{m}}\left(\AA^{3} / \mathrm{Da}\right) / \%$ solvent & $2.63 / 53$ & $2.62 / 53$ & $2.62 / 53$ & $2.61 / 53$ \\
\hline maximum resolution $(\AA)$ (last shell) & $2.20(2.32-2.20)$ & $2.05(2.16-2.05)$ & $2.50(2.63-2.50)$ & $2.60(2.74-2.60)$ \\
\hline no. of unique reflections & 46439 & 317883 & 31238 & 27470 \\
\hline$R_{\mathrm{sym}}(\%)$ & $6.0(36.3)$ & $9.4(38.8)$ & $12.0(38.5)$ & $10.4(31.2)$ \\
\hline$I / \sigma(I)$ & $10.0(2.1)$ & $6.2(1.8)$ & $4.2(1.9)$ & $6.1(2.4)$ \\
\hline multiplicity & $3.3(3.2)$ & $3.8(3.7)$ & $3.1(3.0)$ & $5.8(3.6)$ \\
\hline completeness $(\%)$ & $99.5(99.7)$ & $99.9(99.9)$ & $97.9(97.3)$ & $96.9(83.9)$ \\
\hline
\end{tabular}

derivative of Z. mobilis ACM3963/pZY570R30K/R31K after growth in complex medium supplemented with isopropyl 1 -thio- $\beta$-D-galactopyranoside (1 mM). A coupled anioncation exchange chromatography step was used, followed by a separate cation exchange step using the same buffer, $20 \mathrm{mM}$ HEPES/KOH (pH 7.8), as before. PreGFORcontaining fractions were identified by measurement of GFOR enzymatic activity. Fractions were pooled and equilibrated to $40 \mathrm{mM}$ MES/KOH (pH 6.4) by ultrafiltration. The purity of preparations was checked by SDS-polyacrylamide gel electrophoresis and subsequent Coomassie blue staining or Western blot analysis.

Crystallization. Initial crystallization conditions for preGFOR were found by a grid search around the conditions used to obtain crystals of the mature form of GFOR ( 17 , 26), using polyethylene glycol 6000 (PEG6000) and glycerol (as a cryoprotectant) in succinate buffer ( $\mathrm{pH}$ 5.5). These conditions gave thin crystals that diffracted to only $3 \AA$ resolution, however. Improved crystals were obtained by the addition of ammonium sulfate in the range of $200-400 \mathrm{mM}$, which decreased drastically the number of crystals in the drop and led to thicker, better-diffracting crystals $(0.5 \mathrm{~mm}$ $\times 0.5 \mathrm{~mm} \times 0.2 \mathrm{~mm}$ on average). All crystals were grown in hanging drops by mixing equal quantities of protein solution $(20 \mathrm{mg} / \mathrm{mL}$ preGFOR in $40 \mathrm{mM} \mathrm{MES} / \mathrm{KOH}$ buffered at $\mathrm{pH}$ 6.4) and reservoir solution. The preGFOR succ crystals were obtained using a reservoir that comprised $12 \%$ PEG6000, 20\% glycerol, and $210 \mathrm{mM}$ ammonium sulfate, in $100 \mathrm{mM}$ potassium succinate $(\mathrm{pH}$ 5.5). PreGFOR $\mathrm{gll}$ crystals were grown using similar conditions (12\% PEG6000 and $20 \%$ glycerol) but without succinate and with $550 \mathrm{mM}$ ammonium sulfate. PreGFOR sorb $_{\text {crystals were obtained using }}$ a lower protein concentration $(5 \mathrm{mg} / \mathrm{mL})$ and a reservoir that comprised 16\% PEG6000, $100 \mathrm{mM}$ potassium succinate $(\mathrm{pH}$ $6.5), 5 \%$ glycerol, and $1.5 \mathrm{M}$ sorbitol. Attempts to crystallize preGFOR in the presence of high concentrations of glucose were unsuccessful as precipitates formed very readily; the preGFOR $_{\text {glu }}$ crystals were thus obtained by soaking preGFOR $_{\text {succ }}$ crystals for 8 days in mother liquor containing 300 $\mathrm{mM}$ D-glucose.

Data Collection and Processing. All diffraction data sets were collected on a Mar345 image plate detector using X-radiation ( $\lambda=1.5418 \AA$ ) from a Rigaku RU300 rotatinganode generator equipped with double-focusing mirrors. The crystals were flash-frozen and maintained at $100 \mathrm{~K}$ with an Oxford Cryosystem. For the high-resolution data sets (pre$\mathrm{GFOR}_{\text {gll }}$ and preGFOR $\mathrm{succ}_{\text {s }}$, a $0.5^{\circ}$ oscillation was used for each image to avoid overlapped spots, whereas for pre$\mathrm{GFOR}_{\mathrm{glu}}$ and preGFOR $\mathrm{sorb}$, oscillations of $1^{\circ}$ per image were used. All data sets were processed with DENZO (27), and scaling and merging were carried out in the CCP4 Program Suite (28) with SCALA (29). Details are given in Table 1.

Structure Determination and Refinement. The preGFOR ${ }_{\text {succ }}$ crystals proved to be orthorhombic, in space group $P 2{ }_{1} 2_{1} 2$, with two molecules in the asymmetric unit and a Matthews coefficient of $2.35 \AA^{3} / \mathrm{Da}(30)$. The unit cell (Table 1) corresponded to the form I crystals found by Kingston et al. (17) for mature GFOR. The structure was determined by molecular replacement using AMoRe (31), with the mature GFOR monomer as a starting model after removal of the N-terminal arm (residues 1-31 of mature GFOR), the $\operatorname{NADP}(\mathrm{H})$, and all water molecules. A resolution shell of 10-4 $\AA$ was used for this and all subsequent molecular replacement calculations. One unique solution was found above the background with a correlation coefficient of $60.4 \%$, an $R$-factor of $35.0 \%$, and good crystal packing. Several cycles of rigid body refinement in CNS (32) were carried out, in which each domain of each monomer was allowed to move independently. At this stage, SigmaA-weighted maps (33) were calculated to build in the missing parts of the model. The N-terminal arm (residues 53-83 of preGFOR, corresponding to residues $1-31$ of mature GFOR) and the NADP $(H)$ cofactor were clearly visible in both $2 m F_{\mathrm{o}}-D F_{\mathrm{c}}$ and $m F_{\mathrm{o}}-D F_{\mathrm{c}}$ maps and were added to the model, reducing the $R$-factor and $R_{\text {free }}$ to $30.9 \%$ and $31.1 \%$, respectively. Simulated annealing (34) between 10000 and $100 \mathrm{~K}$ was then used to remove bias from the model. Water molecules were added automatically with CNS and visually inspected using the TURBO-FRODO graphics package (35). Only those sites that had good spherical electron density, reasonable $B$-factors, and hydrogen bond partners with appropriate geometry were retained in the model. The same structure determination and refinement protocols were used for the isomorphous preGFOR glu $_{\text {and }}$ preGFOR sorb $_{\text {structures. Full }}$ refinement statistics are given in Table 2.

Although the crystals of preGFOR gll $_{\text {appeared to be }}$ morphologically identical to those of preGFOR succ $_{\text {, pre- }}$

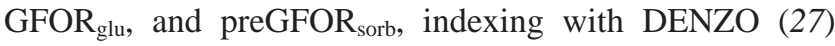
showed that the unit cell was different. Metric tensor distortion indices of $0.15 \%$ for a primitive orthorhombic lattice and $0.09 \%$ for a primitive monoclinic lattice allowed the choice of the higher-symmetry primitive orthorhombic Bravais lattice. The unit cell dimensions ( $a=83.8 \AA, b=$ $279.1 \AA$, and $c=115.7 \AA$ ) corresponded to those of the form II crystals of mature GFOR (17), consistent with six molecules (three dimers) in the asymmetric unit, for a Matthews coefficient of $2.35 \AA^{3} / \mathrm{Da}$. Systematic absences did not allow a unique choice of space group, but molecular replacement with $\mathrm{AMoRe}$, using the preGFOR succ $_{\text {dimer }}$ as a search model, gave a single unique solution in space group 


\begin{tabular}{|c|c|c|c|c|}
\hline \multirow[b]{2}{*}{ resolution limits $(\AA)$} & \multicolumn{4}{|c|}{ preGFOR $_{\text {succ }}$ preGFOR gll preGFOR glu $_{\text {preGFOR }}$ sorb } \\
\hline & $15.0-2.20$ & $15.0-2.05$ & $15.0-2.50$ & $15.0-2.60$ \\
\hline $\begin{array}{l}R \text {-factor (no. of } \\
\text { reflections) }\end{array}$ & $\begin{array}{l}0.215 \\
(45209)\end{array}$ & $\begin{array}{l}0.197 \\
(316326)\end{array}$ & $\begin{array}{l}0.204 \\
(30114)\end{array}$ & $\begin{array}{l}0.200 \\
(26637)\end{array}$ \\
\hline $\begin{array}{l}R_{\text {free }} \text { (no. of } \\
\quad \text { reflections) }\end{array}$ & $\begin{array}{l}0.267 \\
(1382)\end{array}$ & $\begin{array}{l}0.228 \\
(1557)\end{array}$ & $\begin{array}{l}0.275 \\
(931)\end{array}$ & $\begin{array}{l}0.252 \\
(806)\end{array}$ \\
\hline \multicolumn{5}{|l|}{ rms deviation $^{a}$} \\
\hline bond lengths $(\AA)$ & 0.007 & 0.006 & 0.008 & 0.008 \\
\hline \multicolumn{4}{|l|}{ model } & 1.4 \\
\hline $\begin{array}{l}\text { no. of protein } \\
\text { atoms }\end{array}$ & 5920 & 35555 & 5920 & 5920 \\
\hline no. of cofactors & $2 \mathrm{NADP}^{+}$ & $12 \mathrm{NADP}^{+}$ & $2 \mathrm{NADPH}$ & $2 \mathrm{NADPH}$ \\
\hline no. of ligands & 2 succ, 2 glyc & 12 glyc & 0 & 2 glyc \\
\hline $\begin{array}{l}\text { no. of water } \\
\text { molecules }\end{array}$ & 463 & 3952 & 300 & 298 \\
\hline \multicolumn{5}{|l|}{ average $B$-factor $\left(\AA^{2}\right)$} \\
\hline main chain & 38.8 & 21.8 & 46.2 & 36.2 \\
\hline side chain & 40.1 & 23.0 & 47.3 & 36.8 \\
\hline $\begin{array}{l}\text { residues in most } \\
\quad \text { favored region (\%) }\end{array}$ & 89.4 & 89.7 & 87.2 & 89.1 \\
\hline
\end{tabular}

$P 22_{1} 2$, with a correlation coefficient of $66.4 \%$ and an $R$-factor of $42.0 \%$. The next best solution had values of $47.6 \%$ and $49.4 \%$, respectively, and other possible orthorhombic space groups gave significantly worse results. The solution gave good crystal packing, with crystallographic and noncrystallographic symmetry elements generating the expected tetramers. Attempts to refine this structure using rigidbody refinement followed by simulated annealing in CNS (32) or the maximum likelihood protocol in REFMAC (36) failed to reduce the free $R$-factor to $<40 \%$, however. SigmaA-weighted maps (31) did not show any problem in model building, and we were forced to consider alternative explanations. A Patterson calculation indicated that the three dimers in the asymmetric unit would all have the same orientation, related by $1 / 3$ translation along the long axis. However, the reflections along this axis did not show obvious extinction conditions corresponding to a helicoidal axis. This led us to question whether there was indeed a $2_{1}$ axis along this cell edge, and whether the Bravais lattice might be monoclinic rather than orthorhombic, i.e., that not only had the original $b$ axis been tripled but it also no longer had exact screw symmetry.

After the preGFOR ${ }_{\mathrm{gll}}$ data in space group $P 2_{1}$ had been reprocessed (Table 1), molecular replacement in AMoRe gave a unique solution, with a correlation coefficient of $72.3 \%$ and an $R$-factor $37.3 \%$, the next best solution having values of $61.4 \%$ and $43.7 \%$, respectively. The crystal packing was good with the 12 molecules generating three independent tetramers. Rigid body refinement in CNS, with each domain of each monomer allowed to move independently, was followed by simulated annealing between 10000 and $100 \mathrm{~K}$ to remove bias from the model. At this stage, a visual inspection of the model and its SigmaA-weighted maps with TURBO-FRODO allowed some minor errors in the side chain orientations to be corrected. Water molecules were added with ARP/wARP version 5.1 (37), but only those with good $2 m F_{\mathrm{o}}-D F_{\mathrm{c}}$ spherical density, reasonable $B$-factors, and hydrogen bonds with appropriate geometry were retained in the final model. Final refinement statistics are given in Table 2 .

\section{RESULTS}

Crystal Structures. In this work, we have determined the three-dimensional structure of preGFOR under four different sets of conditions. Two of the crystal structures represent the oxidized GFOR, with $\mathrm{NADP}^{+}$bound; these are the preGFOR $_{\text {succ }}$ and preGFOR gll $_{\text {structures, obtained from }}$ crystals prepared in the presence and absence, respectively, of succinate, from a crystallization medium that also contained ammonium sulfate and glycerol. The other two structures should represent the reduced form of preGFOR.

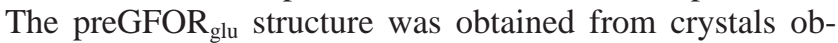
tained in the presence of a high concentration $(0.3 \mathrm{M})$ of glucose, which should cause the reduction of $\mathrm{NADP}^{+}$(Figure $1)$. The preGFOR sorb $_{\text {structure was determined from crystals }}$ prepared from a high concentration $(1.5 \mathrm{M})$ of the product sorbitol which should also favor reduction of $\mathrm{NADP}^{+}$by reversing the second half-reaction (Figure 1). In none of these structures can the 52-residue N-terminal signal peptide be seen, however, except in the preGFOR gll $_{\text {structure where just }}$ the last two residues (51 and 52) are weakly visible. Polyacrylamide gel electrophoresis carried out on several crystals of both preGFOR succ $_{\text {and }}$ aneGFOR gll $_{\text {showed that }}$ the signal sequence had not been proteolyzed during the course of crystallization. In three of the four structures, ligands (glycerol or succinate) were found bound in the active site, and the modeling of these ligands was tested by fitting to "omit" electron density maps (Figure 2) and by refinement.

The crystal structure of preGFOR succ contains two monomers in the asymmetric unit (called A and B) and forms a tetramer by the application of a crystallographic 2-fold axis. It has been refined using data to $2.2 \AA$ resolution and gives an $R$-factor of $21.5 \%$ and an $R_{\text {free }}$ of $26.7 \%$ (see Table 2 for statistics). The final model comprises the whole of the mature GFOR molecule but none of the N-terminal signal peptide. The structure also includes a molecule of succinate in the active site (Figure 2a) and a glycerol molecule at its entry.

Because of the presence of succinate in the catalytic site of the preGFOR ${ }_{\text {succ }}$ structure, we then grew crystals without succinate. Since in this present case the mother liquor was not buffered, we carried out a $\mathrm{pH}$ measurement in the final condition of the drop. The result showed an increase in $\mathrm{pH}$ of 0.5 compared with the previous solutions to which were added $100 \mathrm{mM}$ succinate (pH 5.5). Although this experiment was carried out in the absence of protein, it suggests that the preGFOR ${ }_{\mathrm{gll}}$ crystals were obtained at slightly higher $\mathrm{pH}$, and this may account for the change in space group and tripling of the $c$ axis (Table 1). In this structure, the asymmetric unit contains three tetramers labeled ABCD, EFGH, and IJKL. Although all three tetramers have the same orientation, the translation between tetramer 1 and tetramer 2 is $(-0.00237,0.87637,91.51315 \AA)$ and between tetramer 2 and tetramer 3 is $(6.991394,-0.529892,95.751968 \AA)$; these slight differences explain why the 2-fold screw axis was broken and the $c$ axis tripled. The preGFOR gll $_{\text {structure }}$ has been refined at $2.05 \AA$ resolution, to an $R$-factor of $19.7 \%$ and an $R_{\text {free }}$ of $22.8 \%$ (Table 2). The higher resolution, relative to the other structures, and the slightly different crystallization conditions led us hope that it might be possible to model the signal sequence, but only residue 52 and the backbone of residue 51 in subunit $A$ and residue 52 in subunits $\mathrm{C}-\mathrm{E}, \mathrm{I}$, and $\mathrm{K}$ could be seen. The catalytic site of 

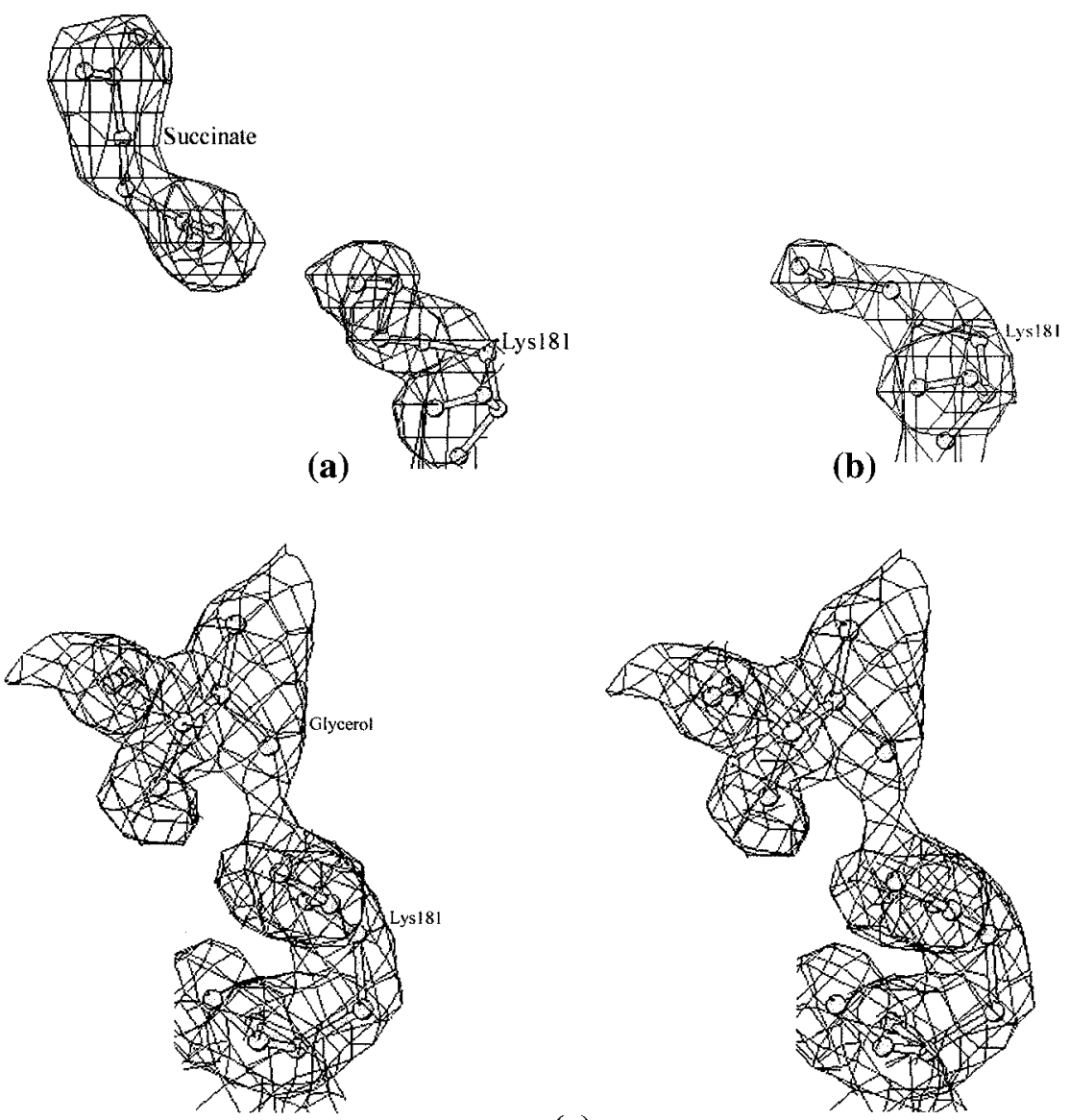

(c)

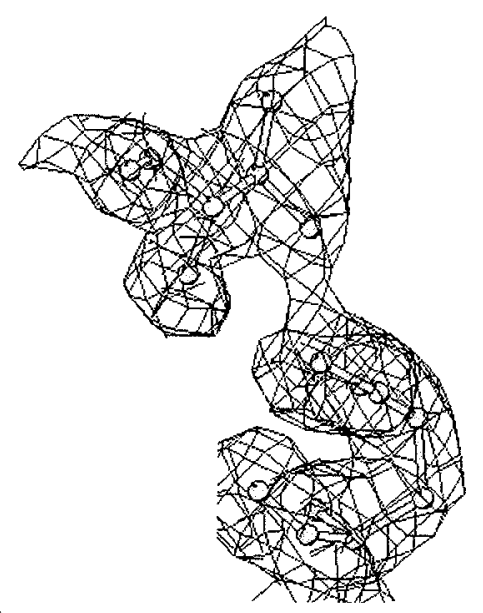

FIGURE 2: Omit electron density showing the bound succinate and glycerol ligands and the conformational change of Lys181. In each case the electron density is contoured at a level of $2 \sigma$. (a) Density for the succinate ion and Lys 181 in preGFOR succ. $_{\text {. }}$ (b) Density for Lys 181 in the preGFOR $_{\text {glu }}$ structure; no ligand is bound in the active site, and Lys181 has changed conformation compared with that in preGFOR succ. (c) Stereoview of the density for glycerol in the active site of preGFOR gll. $_{\text {. }}$ Lys 181 has the same conformation as in preGFOR succ.

preGFOR $\mathrm{gll}_{\mathrm{ll}}$ contains a glycerol molecule (Figure 2c) in place of the succinate present in preGFOR succ. $_{\text {. }}$

The preGFOR $_{\text {glu }}$ and preGFOR sorb strucures were determined at a somewhat lower resolution $(2.5$ and $2.6 \AA$, respectively) with $R$-factors of $20.4 \%$ and $20.0 \%$ and $R_{\text {free }}$ values of $27.5 \%$ and $25.2 \%$, respectively (Table 2). Weak density is present in the catalytic site of preGFOR sorb $_{\text {that }}$ has been modeled as a glycerol molecule, but no convincing density for sorbitol could be seen despite the use of $1.5 \mathrm{M}$ sorbitol in crystallization. PreGFOR glu has an empty catalytic cavity even though the crystal had been soaked in $300 \mathrm{mM}$ glucose.

Overall Molecular Structure. The preGFOR sequence consists of the 381 residues of the mature enzyme preceded by the 52-residue $\mathrm{N}$-terminal signal sequence (18). In the following description, we use residue numbering pertaining to the complete precursor form such that residue 53 corresponds to residue 1 of the mature form. The overall folding of the monomer is identical to that of the mature GFOR structure (17). There are two distinct domains (Figure 3a). The N-terminal domain, residues $84-206$, has the classical dinucleotide binding fold (19). Two $\beta \alpha \beta \alpha \beta$ motifs stack together to form a six-stranded $\beta$-sheet, flanked on either side by $\alpha$-helices. The NADP $(\mathrm{H})$ is bound with its pyrophosphate group oriented toward the $\mathrm{N}$-terminus of the first $\alpha$-helix of the first $\beta \alpha \beta \alpha \beta$ motif and has the same conformation as in the previously determined mature GFOR structure
(17). The adenine ring is in the syn conformation with respect to the glycosidic bond, and the nicotinamide ring is in the anti conformation. The C-terminal domain (residues 207433) consists of nine $\beta$-strands, all antiparallel except for the first one. This $\beta$-sheet is open-faced such that one face is not covered by any loops or helices from its own domain; this face is involved in dimer formation.

$\mathrm{N}$-Terminal residues 1-83 extend from the monomer, although only residues 53-83, which represent the start of the mature enzyme, are visible in the electron density. As in mature GFOR, these residues form an arm that wraps around a neighboring subunit of the tetramer (Figure 3b). The arm conformation is fully extended (it contains seven proline residues) and includes only one short helix (residues 5661). Interactions between this arm and the neighboring subunit, including its $\mathrm{NADP}(\mathrm{H})$ cofactor, begin at residue 59 , meaning that residues $\mathrm{N}$-terminal to this are not restrained by any interaction with the rest of the protein. Moreover, residues 53-51 make a $90^{\circ}$ turn that directs the preceding portion of the signal sequence into a solvent channel in the crystal. The tetramer face from where the signal peptide emerges has a substantial patch of positive surface charge (Figure 4), and this may explain why the signal sequence, which includes a major positively charged portion, does not appear to have a defined structure but is disordered in the solvent channel. 


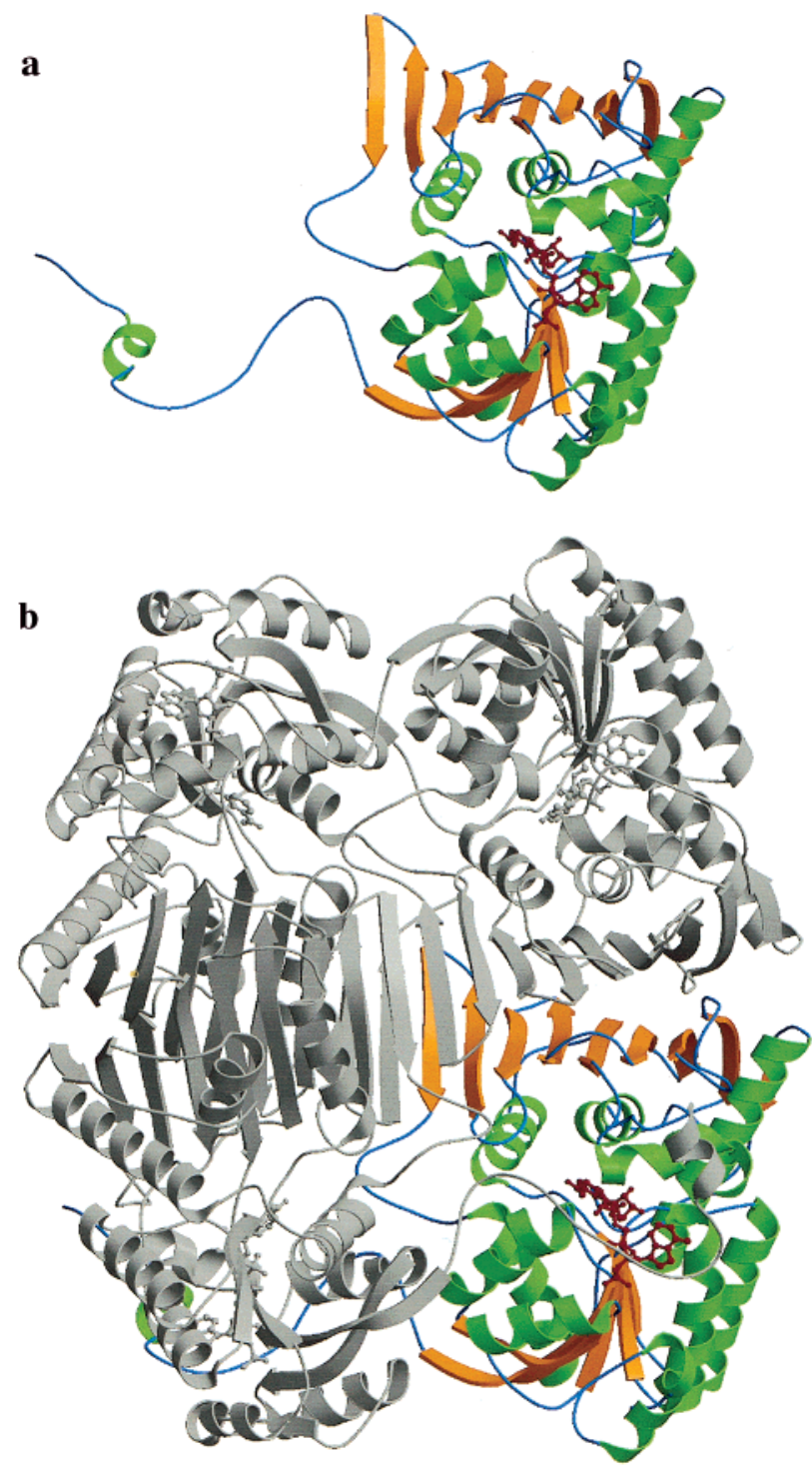

FIGURE 3: Molecular structure of preGFOR. (a) Monomer of preGFOR showing the extended N-terminal arm. The NADP $(\mathrm{H})$ cofactor is shown in a red, ball-and-stick representation. (b) The preGFOR tetramer showing how the $\mathrm{N}$-terminal arm wraps around adjacent monomers. The colored monomer is in the same orientation as in panel a. These and other figures were drawn with MOLSCRIPT (54) and rendered with RASTER3D (55).

Superposition of the various structures also indicates some variability in the initial part of the N-terminal arm. Comparing the preGFOR ${ }_{\text {gll }}$ monomer with that of the mature GFOR structure (17), using as a reference their dinucleotide-binding domains, gives an rms difference in $\mathrm{C} \alpha$ positions of $0.22 \AA$ for the dinucleotide-binding domain, $0.32 \AA$ for the Cterminal domain, and $0.55 \AA$ for the $\mathrm{N}$-terminal arm. Differences in $\mathrm{C} \alpha$ atomic positions between the two structures increase toward the $\mathrm{N}$-terminus of the $\mathrm{N}$-terminal arm, reaching more than $1 \AA$ at residue 53, even though the overall conformation of the arm remains the same. This feature is also seen in comparing preGFOR ${ }_{\text {gll }}$, preGFOR succ $_{\text {, }}$ preGFORsorb, and preGFOR $\mathrm{glu}_{\mathrm{g}}$. The variation is not a consequence of differences in the tetramer structure (see below) but presumably reflects the fact that no interactions stabilize the arm prior to Ala59. The flexibility of the signal peptide may therefore emanate from this point.

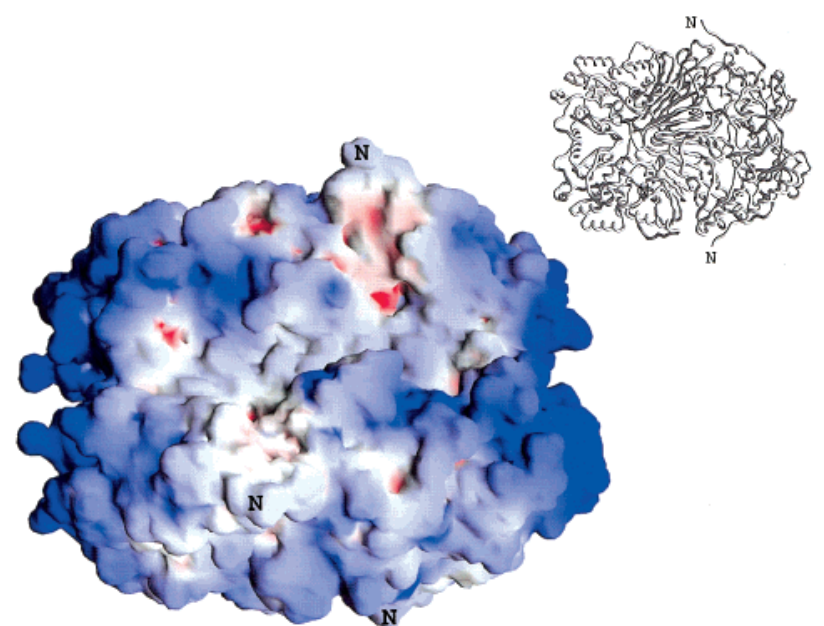

FIGURE 4: Surface of the preGFOR tetramer showing its highly positive character. The orientation of the tetramer is shown by the accompanying worm diagram. The location of the N-terminal residue of the mature enzyme portion (where the signal peptide emerges) is identified with an N. It is proposed that the highly positive surface helps prevent a stable association between the signal peptide and the enzyme.

The preGFOR succ $_{\text {, }}$ preGFOR glu $_{\text {, }}$ and preGFOR sorb $_{\text {structures }}$ all contain a dimer in the crystal asymmetric unit, with the tetramer being completed by application of a crystallographic 2 -fold axis. The preGFOR gll model, on the other hand, comprises three tetramers that have been refined independently (no NCS constraints were used). The root-mean-square (rms) difference in atomic positions between them is never greater than $0.12 \AA$, however, for all main chain atoms, whereas the error in the coordinates is $0.2-0.3 \AA$ (based on a Luzzati plot). This shows that all the tetramers are strictly identical. Superimposing the preGFOR succ $_{\text {tramer on those }}$ of preGFOR gll $_{1}$, preGFOR ${ }_{\text {sorb }}$, and preGFOR glu $_{\text {gives similar }}$ rms differences of $0.23,0.22$, and $0.24 \AA$, respectively. Thus, neither the redox state nor the ligand status changes the overall tetrameric structure.

Like mature GFOR (17), the preGFOR tetramer can be regarded as a dimer of dimers. The surface interaction between the two monomers of the dimer occurs through the open-faced $\beta$-sheet of the $\mathrm{C}$-terminal domain. With a surface area of $3300 \AA^{2}$, it represents $\sim 10 \%$ of the total surface of the monomer. The two dimers then interact mainly via the last strand of the open-faced $\beta$-sheet, creating an 18-stranded $\beta$-sheet with a marked right-handed twist. The $\mathrm{N}$-terminal arm exchange increases drastically the contact surface between dimers and stabilizes the tetramerization such that when this arm is deleted only the dimer is formed (21).

Comparisons with Glucose-6-Phosphate Dehydrogenase. Sequence comparisons show that a number of homologues of GFOR exist $(17,38)$. Three-dimensional structures for three structurally homologous enzymes are known, i.e., dihydrodipicolinate reductase (39), biliverdin reductase (40), and glucose-6-phosphate dehydrogenase (G6PD) $(41,42)$. These oxidoreductases all have the same fold, with a conserved secondary structural core, but with differences in their peripheral features. G6PD has a very strong functional relationship with GFOR, in addition to its structural homology, as it carries out the $\mathrm{NAD}^{+}$- or $\mathrm{NADP}^{+}$-dependent oxidation of glucose 6-phosphate to 6-phosphogluconolactone, a reaction essentially identical to the first half-reaction 
catalyzed by GFOR $(16,18)$. The structure of a ternary complex of a mutant of Leuconostoc mesenteroides G6PD has recently also been determined (43), and we have therefore carried out a detailed superposition of the preGFOR and G6PD structures.

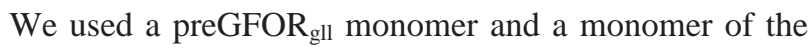
ternary complex of the D177N mutant of L. mesenteroides G6PD, with NADP $(\mathrm{H})$ and glucose 6-phosphate bound (PDB entry 1e7y) for the superposition. Using the program MAPS (44), the secondary structure elements were first identified and aligned, after which the alignment of the two structures was optimized in an iterative process based on both structure and sequence, in which residues were discarded where their displacement was greater than $3 \sigma$, or added to give more extensive alignment. Using this automated process, 148 residues are equivalent with an rms difference in $\mathrm{C} \alpha$ positions of $1.84 \AA$, and the level of sequence identity is $\sim 6 \%$. The structural equivalences extend over both domains of the two proteins; the EKP motifs, which are conserved through this whole family (17), are aligned, and His240, which is the essential acid-base catalytic residue in G6PD (43), aligns precisely with Tyr269, the presumed acid-base catalyst in GFOR (17; see also below).

Ligand Binding in the PreGFOR Catalytic Site. Kinetic studies on preGFOR have shown that the precursor form is fully active and that processing is not a prerequisite for proper folding or catalytic activity $(9,45)$. The redox activity of the enzyme depends on hydride transfer to and from the nicotinamide ring of NADP $(\mathrm{H})$. This ring is almost completely buried inside the protein between the nucleotide binding domain and the $\mathrm{C}$-terminal domain, with only one edge exposed. The crevice housing the cofactor is for the most part buried by the $\mathrm{N}$-terminal arm, with just a small, solvent-filled cavity adjacent to the nicotinamide ring, opening to the outside via an entry $\sim 8 \AA$ in diameter. On one side of the nicotinamide ring is the side chain of Tyr94, which packs against the nicotinamide ring in a plane-to-plane manner (Figure 4). On the other side, where the solventfilled cavity is, are two residues, Tyr269 and Lys181, that have been suggested (17) to have a role in catalysis, analogous to the catalytic Tyr $\cdots$ Lys pairs in enzymes from the aldo-keto reductase (46) and short-chain dehydrogenase (47) families. By analogy with these families, Tyr269 has been proposed to be the essential acid-base catalyst, with Lys181 serving to depress the $\mathrm{p} K_{\mathrm{a}}$ of the Tyr269 hydroxyl group. Mutagenesis supports a catalytic role for both residues, since the Tyr269Phe and Lys181Ala mutants are enzymatically inactive (D. Halbig and G. A. Sprenger, unpublished results). Tyr269 also matches His240, the acid-base catalyst in G6PD, when the preGFOR and G6PD structures are superimposed (see above), and it aligns with Tyr180, which has been shown by mutagenesis to be critical for catalysis in the homologous mammalian dimeric dihydrodiol dehydrogenases (DDs) (48).

Both the bound succinate in preGFOR succ $_{\text {and the bound }}$ glycerol in preGFOR $\mathrm{R}_{\mathrm{gll}}$ bind in the cavity described above, adjacent to the nicotinamide ring and to Tyr269 and Lys 181 (Figure 5a). The glycerol molecule present in preGFOR binds at the bottom of the cavity, hydrogen bonded to Asp265, Arg252, Lys181, and Tyr269. These are all likely candidates for being substrate-binding residues. Asp265 interacts with both $\mathrm{O} 2$ and $\mathrm{O} 3$ of glycerol (both distances of $2.7 \AA), \operatorname{Arg} 252$ with $\mathrm{O} 2(3.0 \AA)$, Lys181 with O1 (2.6 $⿱$ ) and O2 (3.2 $\AA$ ), and Tyr269 with O1 (2.8 $)$ ). Despite these multiple interactions, the glycerol molecule has somewhat higher $B$-factors (average of $24.4 \AA^{2}$ ) than the surrounding structure (average of $13.1 \AA^{2}$ for the nicotinamide ring, for example) and shows evidence of disorder in the $\mathrm{O} 1$ position; we conclude that this is because the glycerol molecule is occupying a space that is designed to accommodate rather larger sugar molecules such as glucose, fructose, and sorbitol; glycerol can be regarded as approximately half of a glucose molecule. The cavity is otherwise filled with water molecules that are interconnected by an extensive hydrogen bonding network. These water molecules must be displaced when a substrate enters.

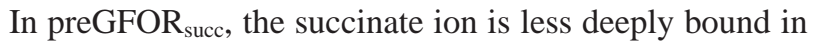
the cavity than is the glycerol in preGFOR gll (Figure 5a). One carboxylate group packs against the nicotinamide and interacts with it via $\pi$-stacking as Tyr94 does on the other side of the ring. The carboxylate oxygens of this carboxylate are also hydrogen bonded to Tyr269 (2.8 ̊), Lys181 (3.4 $\AA$ ), and two water molecules. The second carboxylate is also involved in $\pi$-stacking, with the phenyl ring of Phe369 from an adjacent monomer, and hydrogen bonds with several water molecules. Perhaps because of the nature of these interactions, the succinate is somewhat better defined than is the glycerol in preGFOR gll, and its average $B$-factor $\left(36.0 \AA^{2}\right)$ is more comparable with that of the nicotinamide $\left(25.9 \AA^{2}\right)$. The presence of succinate instead of glycerol in the catalytic site induces the rearrangement of the Asp240 side chain that is located halfway out of the cavity. In preGFOR succ $_{\text {, }}$ this side chain is hydrogen bonded to a water molecule that is further hydrogen bonded to Thr327, whereas in preGFOR ${ }_{\text {gll }}$, the Asp240 side chain is rotated $\sim 60^{\circ}$ toward Thr327, and hydrogen bonds directly with it.

The four structures also show an interesting rearrangement of the catalytically important Lys181, which appears to depend on the oxidation state of the cofactor and the presence or absence of bound ligands. In the two oxidized structures, preGFOR $_{\text {succ }}$ and preGFOR gll $_{\text {gl }}$, the $\epsilon$-amino group of Lys 181 is positioned $4.4 \AA$ from the plane of the nicotinamide ring of the $\mathrm{NADP}^{+}$molecule and $4.3 \AA$ from Tyr269 OH, and the side chain as a whole has an uncommon conformation (where $\mathrm{X}_{1}=40^{\circ}, \mathrm{X}_{2}=60^{\circ}, \mathrm{X}_{3}=60^{\circ}$, and $\mathrm{X}_{4}=180^{\circ}$ ). In this position, Lys $181 \mathrm{~N} \xi$ hydrogen bonds to the main chain carbonyl oxygen of Asp265 and to the $\mathrm{O} 1$ and $\mathrm{O} 2$ hydroxyl oxygens of glycerol (in preGFOR ${ }_{\mathrm{gll}}$ ) or to a carboxylate

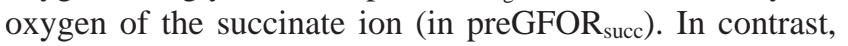
in the two reduced structures, preGFOR ${ }_{\text {glu }}$ and preGFOR ${ }_{\text {sorb }}$, the Lys181 side chain changes its conformation (see, for example, Figure $2 \mathrm{~b}$ ). In preGFOR ${ }_{\mathrm{glu}}$, in which no ligand is bound, the $\epsilon$-amino group of Lys 181 moves $1.0 \AA$ closer to the nicotinamide ring, to a distance of $3.4 \AA$, and closer also to Tyr269 OH $(3.7 \AA)$, as shown in Figure 5b. This closer approach may be stabilized by the more negative charge on the reduced NADPH ring. In preGFOR ${ }_{\text {sorb }}$, where a glycerol molecule is present, Lys181 has the same conformation as in preGFOR glu but does not approach the nicotinamide ring as closely.

\section{DISCUSSION}

Signal Peptide Structure. The signal peptides that target proteins for translocation across membranes and the systems 
a

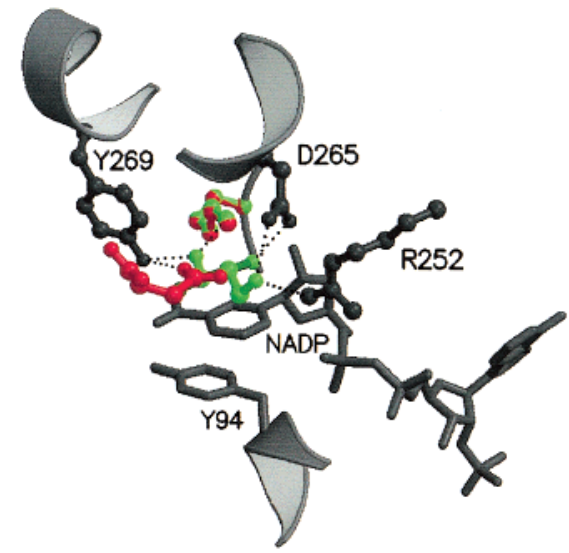

b

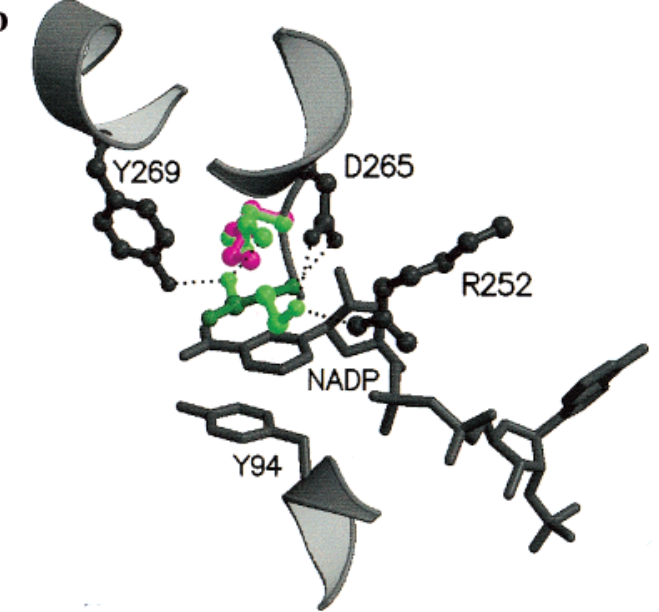

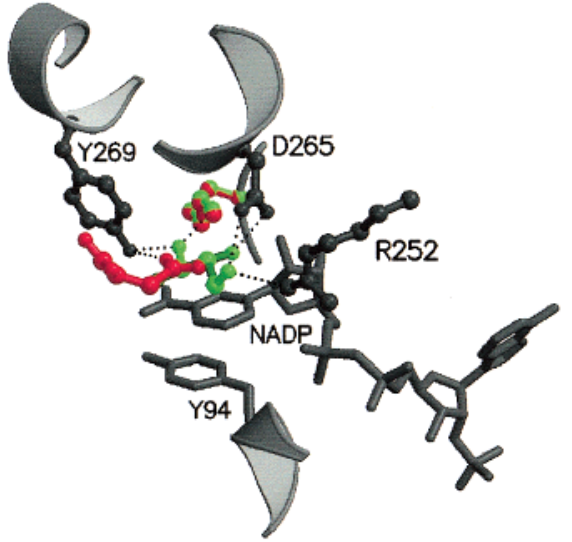

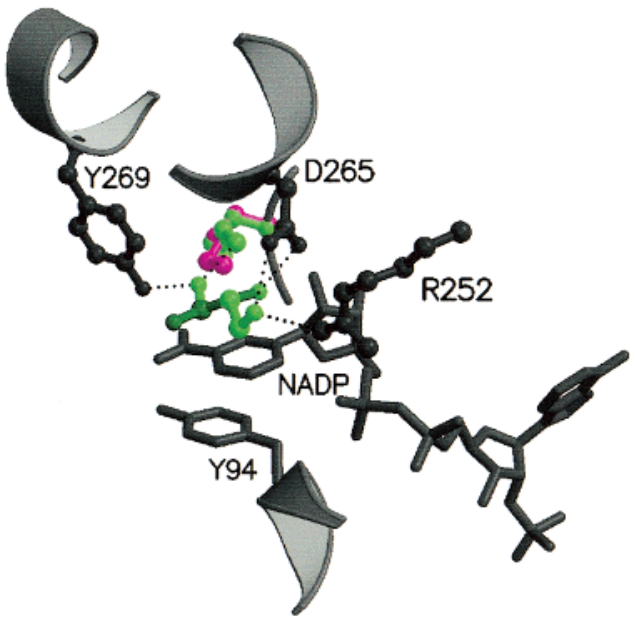

FIGURE 5: Active site region of preGFOR. Changes between the different preGFOR structures are shown in color against the common framework of the preGFOR $\mathrm{gll}_{\mathrm{s}}$ structure (gray). The cofactor structure and the protein structure otherwise are unchanged in the various structures. (a) Stereofigure showing the binding sites for glycerol (green) and succinate (red) in the cavity adjacent to the nicotinamide ring of preGFOR. Arg252, Asp265, and Tyr269, which hydrogen bond to glycerol, and probably also sugar substrates, are shown, together with Tyr94, which packs against the nicotinamide ring. Lys181, which also hydrogen bonds to the glycerol, but whose conformation is sensitive to ligand binding and redox state (see the text), is shown in red (succinate structure) and green (glycerol structure). (b) Stereofigure showing the movement of the Lys181 side chain between the preGFOR gll $_{1}$ structure (green conformation) and the preGFOR glu $_{\text {structure }}(\mathrm{magenta}$

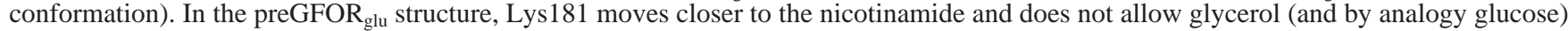
binding in this site (see the text). The glycerol shows disorder at $\mathrm{C} 1$, with the two positions of $\mathrm{O} 1$ shown in light green and dark green.

that mediate these processes have a difficult problem of molecular recognition to overcome; a single translocation apparatus must be able to recognize signal sequences from many different proteins, sharing little sequence homology. In the best-characterized translocation systems, the Secdependent pathway in eubacteria and the signal recognition particle (SRP)-dependent pathway in eukaryotes, the proteins are translocated in an unfolded state (3). Not unreasonably, the signal peptides in these cases are generally regarded as also being unfolded, although there is evidence that they may assume a helical conformation in the hydrophobic environment of a membrane (49). In these cases, it may be the conformational propensity of the signal peptide that matters most, since many different signal peptides must be able to adapt to a common binding environment; in the SRP. this is a hydrophobic groove (50).

The system that is used to translocate GFOR from the cytoplasm into the periplasm of $Z$. mobilis is radically different in several respects. First, the protein is translocated in fully folded form with its redox cofactor $\operatorname{NADP}(\mathrm{H})$ in this case] firmly bound (9). The same system is used to translocate other proteins carrying complex redox cofactors $(6-8)$. Second, the signal peptide is substantially longer, 52 amino acid residues in the case of $\operatorname{GFOR}(15,18)$. This led us to believe that the signal peptide could have a stable folded structure and be presented on the surface of the passenger protein. The ready availability of the precursor form of GFOR has allowed us to test this possibility.

The lack of density for the signal peptide in the crystal structure of preGFOR implies that it is disordered in the crystal. It is possible that signal peptides of this type do not have a defined three-dimensional structure. Alternatively, a hinge prior to residue 53 could allow a (partially) folded signal peptide to explore more than one position in the crystal. In either case, as for the shorter signal peptides associated with Sec-dependent protein translocation, a degree of flexibility in the signal peptide makes good biochemical sense. It could allow it to insert into the translocation apparatus more readily, and it may be essential if many similar but varied sequences are to be recognized by a common template. The signal peptide includes three domains: (i) residues $1-32$, in which the major portion is predominantly polar and/or positively charged; (ii) residues 33-46, which are hydrophobic; and (iii) residues 47-52, which are neutral and mostly polar (9). Flexibility may arise from repulsion between the positively charged domain and 

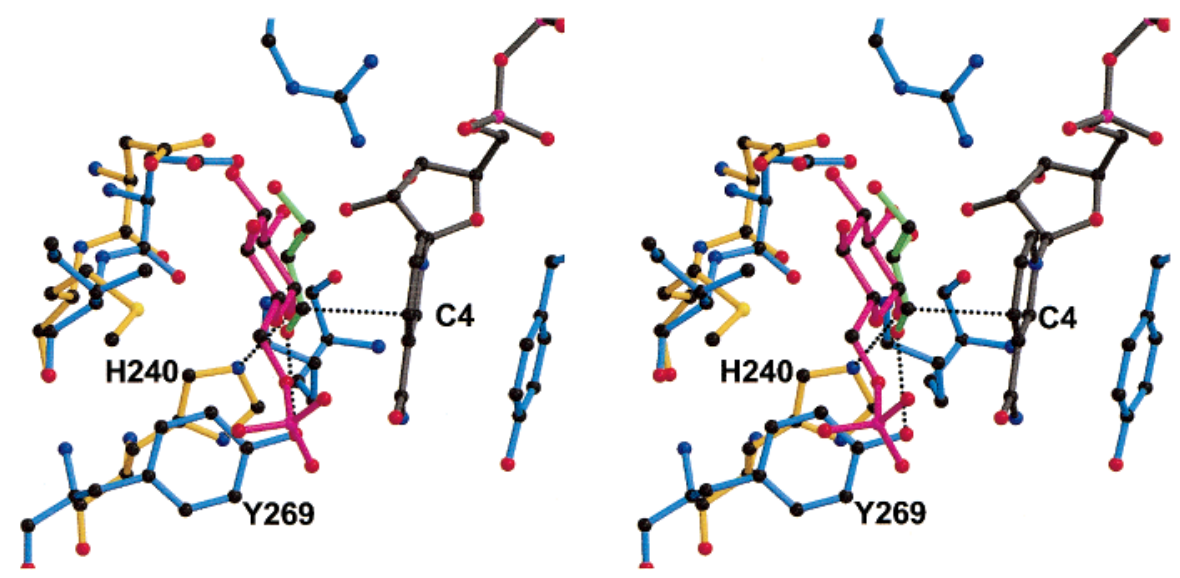

FIGURE 6: Ligand binding in preGFOR and G6PD. The stereofigure shows the active site structure of preGFOR gll $_{\text {[protein, blue; NADP- }}$ $(\mathrm{H})$, gray], with its bound glycerol molecule (green). Key residues of G6PD, i.e., the acid-base catalyst His240, and Asp235 which helps bind the glucose 6-phosphate substrate molecule, are shown in gold in the positions they occupy when the G6PD protein structure is superimposed on preGFOR. Note the correspondence of the glycerol molecule in preGFOR $\mathrm{oll}_{\mathrm{l}}$ with the glucose 6-phosphate molecule (magenta) in G6PD; a small translation would align them perfectly. Note also the correspondence of Tyr269, the acid-base catalyst in preGFOR, with His240, which has the corresponding role in G6PD. Broken lines indicate the close approach of the C1 atom of glycerol to C4 of the nicotinamide ring, and of the $\mathrm{O} 1$ atom of glycerol and glucose 6-phosphate to the Tyr269 OH and His240 NE2 groups, respectively.

the positive charge of the preGFOR surface where the signal peptide emerges; the biggest positive patch is located on the tetramer face where the signal peptide ends (Figure 4). Alternatively, it could result from the presence of six glycine residues in the last two domains of the peptide. We cannot exclude the possibility that disorder of the signal peptide in the present case is a consequence of crystal packing forces, i.e., that the crystal packing is dictated by the rest of the preGFOR molecule and does not allow the signal peptide to take up a single defined structure. Even this would imply flexibility, however, as crystal packing forces are generally very weak.

Substrate Binding and Catalysis. A remarkable feature of GFOR activity is that one substrate in the ping-pong reaction, $\operatorname{NADP}(\mathrm{H})$, is extremely tightly bound such that it does not dissociate during the reaction cycle, but that the affinities for the other two substrates, glucose and fructose, are extremely low $\left[K_{\mathrm{m}}\right.$ for glucose $>10.8 \pm 0.8 \mathrm{mM} ; K_{\mathrm{m}}$ for fructose $>400 \pm 30 \mathrm{mM}$ (12)]. The sugar substrates use the same site (12), implying that although the binding site is quite specific for the $\beta$-anomer of D-glucose over the $\alpha$-anomer, it must also be able to accommodate the linear form of D-fructose for reduction to D-sorbitol. This may account for the low affinity for sugar substrates. It also means that the formation of sorbitol as an osmoprotectant is restricted to conditions when the fructose concentration is high (maximum activity around $1 \mathrm{M}$ fructose). Although kinetic and mechanistic studies have all been carried out on the mature form of $\operatorname{GFOR}(11,12)$, both the wild-type preGFOR and its R30K/R31K mutant form are enzymatically active $(9,45)$, and the results presented here show that the structure in and around the active site of preGFOR is identical to that of mature GFOR. Our observations of ligand binding to preGFOR should therefore be relevant to the mature enzyme as well.

The site occupied by glycerol and by succinate in the crystal structures of preGFOR gll $_{\text {and }}$ aneGFOR succ $_{\text {, }}$, respectively, almost certainly represents the sugar binding site of GFOR. Both ligands bind adjacent to the nicotinamide ring in a shallow, water-filled cleft that opens to the surface. Both are also hydrogen bonded to the $\mathrm{OH}$ group of Tyr269. There is little doubt that Tyr269 is the essential acid-base catalyst in GFOR. Mutation of Tyr269 to Phe abolishes activity (D. Halbig and G. A. Sprenger, unpublished results); it is structurally homologous with His240, the acid-base catalyst in the related enzyme G6PD (43), and it corresponds with Tyr180, which is critical for catalysis in the homologous dimeric DDs (48).

The glycerol binding mode in preGFOR $\mathrm{R}_{\mathrm{gll}}$ appears to be especially significant. Glycerol is a common competitive inhibitor of sugar binding, and when the structure of a ternary complex of G6PD (43) is superimposed onto preGFOR $\mathrm{gll}_{\mathrm{gl}}$, the glycerol molecule in the latter is seen to match perfectly with atoms $\mathrm{C} 1, \mathrm{O} 1, \mathrm{C} 2, \mathrm{O} 2, \mathrm{C} 3$, and $\mathrm{O} 3$ of the glucose 6-phosphate molecule in the former (Figure 5), in both position and conformation. In the standard mechanism for the oxidation of glucose, the catalytic base (here assumed to be the phenolate oxygen of Tyr269) removes a proton from the $\mathrm{C} 1 \mathrm{OH}$ group of glucose, and $\mathrm{NADP}^{+}$abstracts a hydride ion $\left(\mathrm{H}^{-}\right)$from the $\mathrm{C} 1$ atom. In the glycerol complex, preGFOR $_{\text {gll }}$, the $\mathrm{O} 1$ hydroxyl group of glycerol is hydrogen bonded $(2.8 \AA)$ to the Tyr269 OH group, in perfect position for the proton abstraction by Tyr269. The $\mathrm{C} 1$ atom is also only $2.9 \AA$ from the $\mathrm{C} 4$ atom of the nicotinamide ring, the hydride ion acceptor (Figure 6). This binding mode therefore almost certainly mimics the productive binding mode of $\beta$-Dglucose in GFOR. Additionally, the $\mathrm{O} 2$ and $\mathrm{O} 3$ hydroxyl groups of glycerol are hydrogen bonded ( $2.7 \AA)$ to the OD1 and OD2 carboxylate oxygens of Asp265. In G6PD, the structurally homologous residue Asp235 makes equivalent interactions with the glucose 6-phosphate substrate (43), and in the DDs, the equivalent Asp176 is also implicated in substrate binding (51). Therefore, we conclude that Asp265 has an important role in the binding of the glucose substrate in GFOR. The other groups involved in glycerol binding in preGFOR $_{\text {gll }}$ Lys181 and Arg252 [which also hydrogen bonds to Asp265 and an $\operatorname{NADP}(\mathrm{H})$ phosphate oxygen at one end of the cavity], should also have a role in glucose binding.

The role of Lys181 in the catalytic mechanism is of particular interest. This residue is part of a conserved EKP motif that is present in all enzymes of the GFOR family. It lies below the sugar binding site (it is buried by the bound 
glycerol molecule) with its $\epsilon$-amino group $\sim 4 \AA$ from the OH group of Tyr 269 and $4 \AA$ from the nicotinamide ring of the cofactor. Its obvious functional role is to reduce the $\mathrm{p} K_{\mathrm{a}}$ of the Tyr269 $\mathrm{OH}$ group so that the latter can act as the catalytic acid-base catalyst, creating a Tyr $\cdots$ Lys catalytic diad as in the short-chain dehydrogenases (47) and aldoketo reductases (46). The present structures show, however, that the Lys181 side chain position and conformation are sensitive to the oxidation state of the cofactor and the presence or absence of ligands. The conformation of Lys 181 changes between the two putative oxidized structures, preGFOR $_{\text {succ }}$ and preGFOR gll $_{\text {, }}$, and the two putative reduced structures, preGFOR ${ }_{\mathrm{glu}}$ and preGFOR ${ }_{\text {sorb. }}$. Moreover, in the preGFOR $_{\text {glu }}$ structure, the only one without a ligand bound, it moves closer to the nicotinamide ring such that its $\epsilon$-amino group is only 3.3-3.4 $\AA$ from the nicotinamide ring, compared with $4.4-4.6 \AA$ in the oxidized structures (Figure $5 b)$. In this position, Lys181 NZ would inhibit glycerol or glucose binding as it is less than $2 \AA$ from the substrate $\mathrm{O} 1$ position.

The movement of Lys181 seen in preGFOR glu $_{\text {suggests }}$ an additional role for this residue, preventing the rebinding of glucose when the cofactor is in its reduced state by moving closer to the nicotinamide ring. This undoubtedly explains why neither glucose nor glycerol is bound in the structure

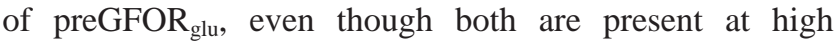
concentrations. Mechanistic studies of GFOR show that the lactone product of glucose oxidation triggers a conformational change that can ultimately lead to enzyme inactivation; this conformational change can be detected by an increased susceptibility of the NADPH cofactor to fluorescence quenching by iodide (52). Our structural results strongly suggest that the movement of Lys181 closer to the nicotinamide ring is responsible for this phenomenon. This movement only occurs in the glucose-soaked crystals where oxidation of glucose to gluconolactone can be expected to have occurred; it does not occur in the sorbitol-soaked crystals. Movement of the Lys181 $\epsilon$-amino group closer to the nicotinamide ring could lead to binding of iodide ions closer to the ring, with resulting fluorescence quenching.

We conclude that the Lys181 side chain plays a critical role in the reaction cycle of GFOR. Glucose binds in the site occupied by glycerol in our preGFOR gll $_{\text {chystals, }}$ hydrogen bonded to Tyr269 and (among other residues) Lys181. Oxidation of glucose to the lactone product results in a movement of Lys181 so that its $\epsilon$-amino group is $\sim 1 \AA$ closer to the nicotinamide ring. It is stabilized in this position by the increased negative charge on the reduced nicotinamide moiety. In this position it also prevents the rebinding of glucose by partly occupying the glucose binding site. This ensures that GFOR does not act as a glucose dehydrogenase. Only under conditions with a high fructose concentration does the second half-reaction occur, using the reduced NADPH to produce sorbitol from fructose. These are the physiological conditions under which sorbitol production is required, for osmoprotection.

\section{REFERENCES}

1. Pugsley, A. P. (1993) Microbiol. Rev. 57, 50-108.

2. Driessen, A. J. M. (1994) J. Membr. Biol. 142, 145-159.

3. Randall, L. L., and Hardy, S. L. S. (1986) Cell 46, 921-928.
4. Chaddock, A. M., Mant, A., Karnauchov, I., Brink, S., Herrmann, R. G., Klösgen, R. B., and Robinson, C. (1995) EMBO J. 14, 2715-2722.

5. Robinson, C., and Mant, A. (1997) Trends Plant Sci. 2, 431437.

6. Berks, B. C. (1996) Mol. Microbiol. 22, 393-404.

7. Dalbey, R. E., and Robinson, C. (1999) Trends Biochem. Sci. $24,17-22$

8. Weiner, J. H., Bilous, P. T., Shaw, G. M., Lubitz, S. P., Frost, L., Thomas, G. H., Cole, J. A., and Turner, R. J. (1998) Cell 93, 93-101.

9. Halbig, D., Wiegert, T., Blaudeck, N., Freudl, R., and Sprenger, G. A. (1999) Eur. J. Biochem. 263, 543-551.

10. Loos, H., Krämer, R., Sahm, H., and Sprenger, G. A. (1994) J. Bacteriol. 176, 7688-7693.

11. Zachariou, M., and Scopes, R. (1986) J. Bacteriol. 167, 863869.

12. Hardman, M. J., and Scopes, R. (1988) Eur. J. Biochem. 173, 203-209.

13. Strohdeicher, M., Schmitz, B., Bringer-Meyer, S., and Sahm, H. (1988) Appl. Microbiol. Biotechnol. 27, 278-282.

14. Loos, H., Sahm, H., and Sprenger, G. A. (1993) FEMS Microbiol. Lett. 107, 293-298.

15. Kanagasundaram, V., and Scopes, R. (1992) Biochim. Biophys. Acta 1171, 198-200.

16. Wiegert, T., Sahm, H., and Sprenger, G. A. (1996) Arch. Microbiol. 166, 32-41.

17. Kingston, R. L., Scopes, R. K, and Baker, E. N. (1996) Structure 4, 1413-1428.

18. Wiegert, T., Sahm, H., and Sprenger, G. A. (1997) J. Biol. Chem. 272, 13126-13133.

19. Lesk, A. M. (1995) Curr. Opin. Struct. Biol. 5, 775-783.

20. Richardson, J. S. (1981) Adv. Protein Chem. 34, 167-339.

21. Lott, J. S., Halbig, D., Baker, H. M., Hardman, M. J., Sprenger, G. A., and Baker, E. N. (2000) J. Mol. Biol. 304, 575-584.

22. Nurizzo, D., Silvestrini, M. C., Mathieu, M., Cutruzzola, F., Bourgeois, D., Fulop, V., Hajdu, J., Brunori, M., Tegoni, M., and Cambillau, C. (1997) Structure 5, 1157-1171.

23. Bennett, M. J., Schlunegger, M. P., and Eisenberg, D. (1995) Protein Sci. 4, 2455-2468.

24. Yanisch-Perron, C., Vieira, J., and Messing, J. (1985) Gene 33, 103-119.

25. Simon, R., Priefer, U., and Pühler, A. (1983) Bio/Technology 1, 784-790.

26. Loos, H., Ermler, U., Sprenger, G. A., and Sahm, H. (1994) Protein Sci. 3, 2447-2449.

27. Otwinowski, Z., and Minor, W. (1997) Methods Enzymol. 276, $307-326$.

28. Collaborative Computing Project Number 4 (1994) Acta Crystallogr. D50, 760-763.

29. Evans, P. R. (1993) Proceedings of CCP4 Study Weekend, pp 114-122, Daresbury Laboratory, Warrington, U.K.

30. Matthews, B. W. (1968) J. Mol. Biol. 33, 491-497.

31. Navaza, J. (1994) Acta Crystallogr. A50, 157-163.

32. Brünger, A. T., Adams, P. D., Clore, G. M., DeLano, W. L., Gros, P., Grosse-Kunstleve, R. W., Jiang, J.-S., Kuszewski, J., Nilges, M., Pannu, N. S., Read, R. J., Rice, L. M., Simonson, T., and Warren, G. L. (1998) Acta Crystallogr. D54, 905-921.

33. Read, R. J. (1990) Acta Crystallogr. A46, 140-149.

34. Brünger, A. T., Kuriyan, J., and Karplus, M. (1987) Science $235,458-460$.

35. Roussel, A., and Cambillau, C. (1991) in Silicon Graphics Geometry Partners Directory, p 81, Silicon Graphics Corp.

36. Murshudov, G. N., Vagin, A. A., and Dodson, E. J. (1997) Acta Crystallogr. D53, 240-255.

37. Perrakis, A., Sixma, T. K., Wilson, K. S., and Lamzin, V. S. (1997) Acta Crystallogr. D52, 448-455.

38. Arimitsu, A., Aoki, S., Ishikura, S., Nakanishi, S., Matsuura, K., and Hara, A. (1999) Biochem. J. 342, 721-728.

39. Scapin, G., Reddy, S. G., and Blanchard, J. S. (1997) Biochemistry 36, 15081-15088. 
40. Kikuchi, A., Park, S.-Y., Miyatake, H., Sun, D., Sato, M., Yoshida, T., and Shiro, Y. (2001) Nature Struct. Biol. 8, 221225.

41. Rowland, P., Basak, A. K., Gover, S., Levy, H. R., and Adams, M. J. (1994) Structure 2, 1073-1087.

42. Au, S. W. N., Gover, S., Lam, V. M. S., and Adams, M. J. (2000) Structure 8, 293-303.

43. Cosgrove, M. S., Gover, S., Naylor, C. E., Vandeputte-Rutten, L., Adams, M. J., and Levy, H. R. (2000) Biochemistry 39, $15002-15011$.

44. Lu, G. (2000) J. Appl. Crystallogr. 33, 176-183.

45. Loos, H., Sahm, H., and Sprenger, G. A. (1993) FEMS Microbiol. Lett. 107, 293-298.

46. Bohren, K. M., Grimshaw, C. E., Lai, C.-J., Harrison, D. H., Ringe, D., Petsko, G. A., and Gabbay, K. H. (1994) Biochemistry 33, 2021-2032.

47. Jörnvall, H., Persson, B., Krook, M., Atrian, S., GonzalezDuarte, R., Jeffery, J., and Ghosh, D. (1995) Biochemistry 34, 6003-6013.
48. Asada, Y., Aoki, S., Ishikura, S., Usami, N., and Hara, A. (2000) Biochem. Biophys. Res. Commun. 278, 333337.

49. Wang, Z., Jones, J. D., Rizo, J., and Gierasch, L. M. (1993) Biochemistry 32, 13991-13999.

50. Keenan, R. J., Freymann, D. M., Walter, P., and Stroud, R. M. (1998) Cell 94, 181-191.

51. Aoki, S., Ishikura, S., Asada, Y., Usami, N., and Hara, A. (2001) Chem.-Biol. Interact. 130-132, 775-784.

52. Fürlinger, M., Haltrich, D., Kulbe, K. D., and Nidetzky, B. (1998) Eur. J. Biochem. 251, 955-963.

53. Engh, R. A., and Huber, R. (1991) Acta Crystallogr. A47, 392-400.

54. Kraulis, P. J. (1991) J. Appl. Crystallogr. 24, 946-950.

55. Merritt, E. A., and Murphy, M. E. P. (1994) Acta Crystallogr. D50, 869-873.

BI011355D 